\title{
Measurement of lifetimes by laser-induced fluorescence and determination of transition probabilities of astrophysical interest in Nd III
}

\author{
Z. G. Zhang ${ }^{1}$, S. Svanberg ${ }^{1}$, P. Palmeri ${ }^{2}$, P. Quinet ${ }^{2,3}$, and E. Biémont ${ }^{2,3}$ \\ 1 Department of Physics, Lund Institute of Technology, PO Box 118, 22100 Lund, Sweden \\ 2 Astrophysique et Spectroscopie, Université de Mons-Hainaut, 7000 Mons, Belgium \\ 3 IPNAS, Bât. B15, Université de Liège, Sart Tilman, 4000 Liège, Belgium
}

Received 04 July 2001 / Accepted 20 December 2001

\begin{abstract}
Selective lifetime measurements by time-resolved laser-induced fluorescence spectroscopy for 5 levels belonging to the $4 \mathrm{f}^{3} 5 \mathrm{~d}$ configuration of doubly ionized neodymium provide a first and useful experimental test of the relativistic Hartree-Fock calculations in this ion of astrophysical interest. As a consequence, the accuracy of the transition probabilities deduced in the present work is well assessed. These new data are expected to help astrophysicists in the future to refine the analysis of the composition of chemically peculiar stars which frequently show large overabundances of lanthanides when compared to the solar system standards.
\end{abstract}

Key words. atomic data - atomic processes - stars: chemically peculiar

\section{Introduction}

Natural neodymium appears in the form of seven stable isotopes. According to nucleosynthesis theories, the isotopes 143 (12.2\%), 144 (23.8\%), $145(8.3 \%)$ and $146(17.2 \%)$ are produced either by the $r$ or $s$ process while the isotope $142(27.1 \%)$ results from the $s$ process and the isotopes $148(5.8 \%)$ and $150(5.6 \%)$ are formed by a pure $r$ process.

Singly-ionized neodymium (Nd II) is frequently observed in astrophysical spectra (Merril 1956; Jaschek \& Jaschek 1995): it has been identified in M and MS stars e.g. by Smith \& Lambert (1985) and in Bp stars e.g. by Cowley \& Crosswhite (1978). Some Nd II lines appear very strongly in the stars of the Cr-Eu-Sr subgroup (Adelman 1973) or in Am stars (Smith 1973, 1974). The analysis of Nd II lines leads to strong overabundances in Ba stars (Lambert 1985) or in S-type stars (Bidelman 1953). $\mathrm{Nd}$ II is also observed in the solar photospheric spectrum (Anders \& Grevesse 1989).

Doubly ionized neodymium (Nd III), which is expected to be the dominant species in hotter stars, has been identified in the spectra of some CP stars like HR 465, HD 51418 and HD 200311 (Cowley 1976; Aikman et al. 1979; Bidelman et al. 1995) and in the spectrum of HD 192913 and HD 43819, Bp stars of the Si subgroup (Cowley \& Crosswhite 1978; Ryabchikova et al. 1990).

Send offprint requests to: E. Biémont, e-mail: E.Biemont@ulg.ac.be
Some Nd III lines have also been found in the spectrum of HD 101065, a rapidly oscillating star also known as Przybylski's star (Cowley et al. 1998, 2000). However, the difficulty of analyzing the third spectrum of most rare earth elements (REE), including Nd III, in low resolution IUE spectra has been discussed by Cowley \& Greenberg (1988) in relation to the uncertainties affecting the solar model and in relation to the lack of reliable $g f$-values. The new high-resolution, high signal-to-noise HST spectra now available (Leckrone et al. 1999) allow the identification of many features belonging to the doubly ionized lanthanides that emphasize the need for accurate radiative parameters for all the REE. These data are particularly important for the study of CP stars that frequently show large overabundances of lanthanides when compared to the solar system values (see e.g. Cowley 1984) and for the metal-poor galactic halo stars in relation to the early history of the galaxy (see e.g. Sneden et al. 1996).

Doubly ionized neodymium (Nd III, $Z=60$ ) belongs to the Ce I isoelectronic sequence and has $[\mathrm{Xe}] 4 \mathrm{f}^{4}{ }^{5} \mathrm{I}_{4}$ as the ground state. Up to now, according to the NIST compilation (Martin et al. 1978; http://physics.nist.gov/cgi-bin/AtData), only 29 levels of this ion have been experimentally determined. They belong to the $4 \mathrm{f}^{4}$ and to the $4 \mathrm{f}^{3}\left({ }^{4} \mathrm{I}^{\circ},{ }^{4} \mathrm{~F}^{\circ}\right) 5 \mathrm{~d}$ configurations. They were deduced from the 9500 lines observed between 181.0 and $871.5 \mathrm{~nm}$ by Crosswhite $(1975,1976)$. This spectrum is thus very poorly known and in need of completion. 
Previous transition probability calculations in Nd III are extremely scarse. To our knowledge, the only available data have been obtained by Cowley \& Bord (1998) who calculated $g f$-values for the analysis of the spectra of $\gamma$ Equulei and HR 6870, and by Bord (2000) who performed HFR calculations having in mind the analysis of the chemically peculiar stars HD 122970 and HD 101065. No experimental measurements (lifetimes or transition probabilities) are available so far for Nd III. In view of the complexity of the configurations involved in the REE, experimental transition probabilities or radiative lifetimes are however indispensable to test the validity of the theoretical models.

The above considerations concerning the need for and the lack of radiative atomic data for the $\mathrm{Nd}^{2+}$ ion justify the present effort to improve the situation. In fact, to meet our objectives, i.e. the determination of a large number of transition probabilities of astrophysical interest as accurately as possible, we have compared laser lifetime measurements for 5 levels with theoretical values resulting from configuration interaction calculations. Although the theoretical approach used was similar to that considered by Bord (2000), the present calculation considers configuration interactions (that are vital in the complex spectrum considered here) in a more detailed way, with the consequence that the deduced transition probabilities are expected to be more accurate.

The present work is part of an extensive program of lifetime measurements in doubly ionized REE carried out at the Lund Laser Centre (LLC) in Sweden and of transition probability calculations at the Liège and Mons universities. So far, the results obtained concern about 50000 transitions of the following ions: La III (Li \& Jiang 1999), La III - Lu III (Biémont et al. 1999), Ce III (Li et al. 2000), Gd III (Zhang et al. 2000), Er III (Biémont et al. 2001a), Pr III (Palmeri et al. 2001; Biémont et al. 2001b), Tm III (Li et al. 2001), Yb III (Biémont et al. 2001c), Ho III (Biémont et al. 2001d) and Eu III (Zhang et al. 2001). These new atomic data are progressively incorporated in a database of astrophysical interest, D.R.E.A.M., which is available on a web site at the address: http://www.umh.ac.be/ astro/dream.shtml and is also accessible directly through anonymous ftp at the address: umhsp02.umh.ac.be/pub/ftp_astro/dream.

\section{Experimental measurements}

The five levels of Nd III, considered in the present experiment, belong to the configuration $4 \mathrm{f}^{3} 5 \mathrm{~d}$ and their lifetimes were measured using two-step excitation timeresolved laser-induced fluorescence technique. The experimental schemes followed for the measurements are summarized in Table 1.

The experimental set-up used in the present experiment is illustrated in Fig. $1 . \mathrm{Nd}^{2+}$ ions were produced in a laser-induced plasma using $532 \mathrm{~nm}$ wavelength laser pulses emitted with a $10 \mathrm{~Hz}$ repetition rate and $10 \mathrm{~ns}$ duration Nd:YAG laser (laser B) (Continuum surelite)

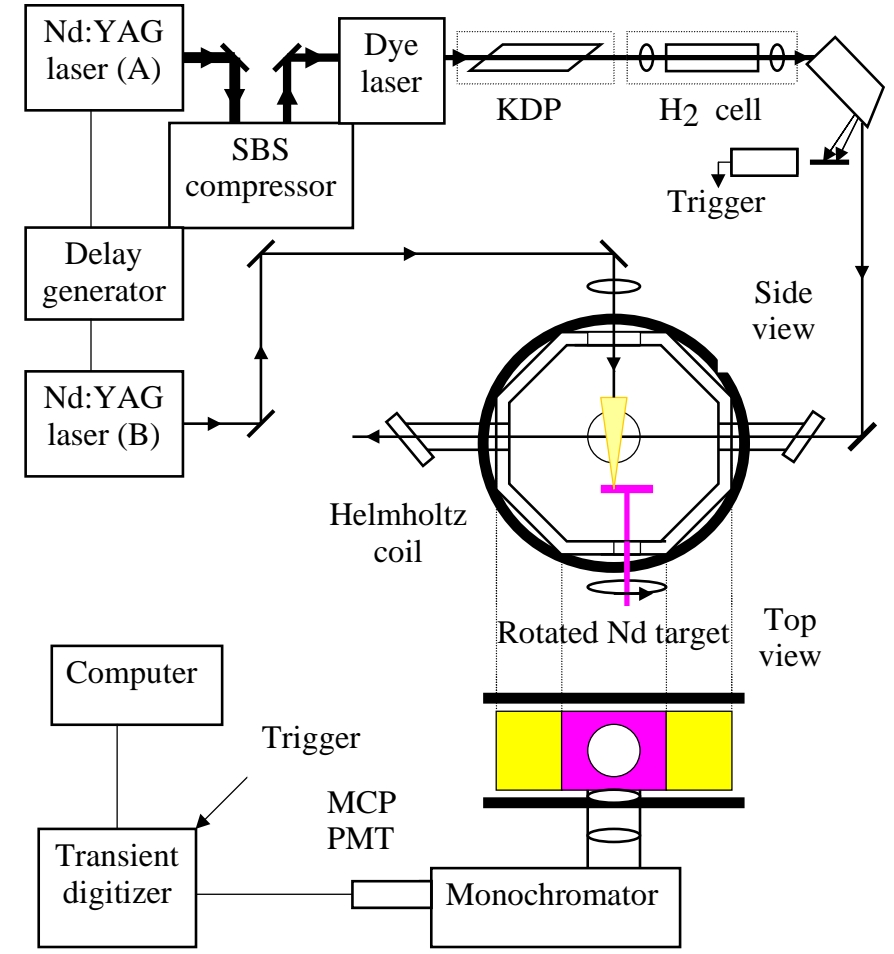

Fig. 1. Experimental set-up used for the lifetime measurements for Nd III.

with variable pulse energy $(0-50 \mathrm{~mJ})$. In order to obtain the suitable excitation, 8 ns pulses emitted by another Nd:YAG laser (laser A) (Continuum NY-82) were sent to a stimulated Brillouin scattering (SBS) compressor to shorten the pulses down to $1 \mathrm{~ns}$. The laser was used to pump a dye laser (Continuum Nd-60). DCM dye was used in the experiments, and the frequency-doubling of the dye laser and further stimulated Raman scattering were obtained using a KDP crystal and a hydrogen cell.

The lasers were triggered by a digital delay generator (Stanford Research System, Model 535) and the delay between the ablation and excitation pulses was adjusted. After the ions were produced through perpendicularly focusing the B laser onto the surface of a Nd foil rotated in a vacuum chamber, the ions were excited selectively by the excitation laser crossing the plasma horizontally. The fluorescence decay was imaged by two $\mathrm{CaF}_{2}$ lenses and concentrated on the entrance slit of a vacuum monochromator. A Hamamatsu R3809U-58 photomultiplier was used for the detection.

The time-resolved signal was averaged with a digital transient recorder (Tektronix model DSA 602) and the fluorescence decay curve was sent to a personal computer for lifetime determination. The lifetime evaluation was performed by fitting the fluorescence decay signal curve with an exponential function with adjustable parameters. For each level, more than 20 curves were averaged. The five lifetimes measured are reported in Table 2.

In order to be sure that the $\mathrm{Nd}^{2+}$ lines of interest were considered, the modification of the fluorescence signal as a function of the delay time was investigated. Possible 
Table 1. Nd III levels measured and the corresponding excitation schemes.

\begin{tabular}{lrrrccc}
\hline Levels & & $\begin{array}{r}\text { Lower level } \\
\left(\mathrm{cm}^{-1}\right)\end{array}$ & $\begin{array}{r}\text { Upper level } \\
\left(\mathrm{cm}^{-1}\right)\end{array}$ & $\begin{array}{r}\text { Excitation } \\
\lambda_{\text {air }}(\mathrm{nm})\end{array}$ & $\begin{array}{c}\text { Laser } \\
\text { mode }^{\mathrm{b}}\end{array}$ & $\begin{array}{c}\text { Observed } \\
\lambda_{\text {air }}(\mathrm{nm})\end{array}$ \\
\hline $4 \mathrm{f}^{3}\left({ }^{4} \mathrm{~F}^{\circ}\right) 5 \mathrm{~d}$ & ${ }^{5} \mathrm{H}_{3}^{\circ}$ & 0.0 & 27788.2 & 359.762 & $2 \omega+S$ & 359.8 \\
$4 \mathrm{f}^{3}\left({ }^{4} \mathrm{~F}^{\circ}\right) 5 \mathrm{~d}$ & ${ }^{5} \mathrm{H}_{4}^{\circ}$ & 1137.8 & 28745.3 & 362.117 & $2 \omega+S$ & 362.1 \\
$4 \mathrm{f}^{3}\left({ }^{4} \mathrm{~F}^{\circ}\right) 5 \mathrm{~d}$ & ${ }^{5} \mathrm{H}_{5}^{\circ}$ & 2387.6 & 30232.3 & 359.032 & $2 \omega+S$ & 359.0 \\
$4 \mathrm{f}^{3}\left({ }^{4} \mathrm{~F}^{\circ}\right) 5 \mathrm{~d}$ & ${ }^{5} \mathrm{H}_{6}^{\circ}$ & 3714.9 & 31394.6 & 361.173 & $2 \omega+S$ & 361.2 \\
$4 \mathrm{f}^{3}\left({ }^{4} \mathrm{~F}^{\circ}\right) 5 \mathrm{~d}$ & ${ }^{5} \mathrm{H}_{7}^{\circ}$ & 5093.3 & 32832.6 & 360.397 & $2 \omega+S$ & 360.4 \\
\hline
\end{tabular}

a From Martin et al. (1978).

b $2 \omega$ means frequency-doubling and $S$ is written for Stokes component.

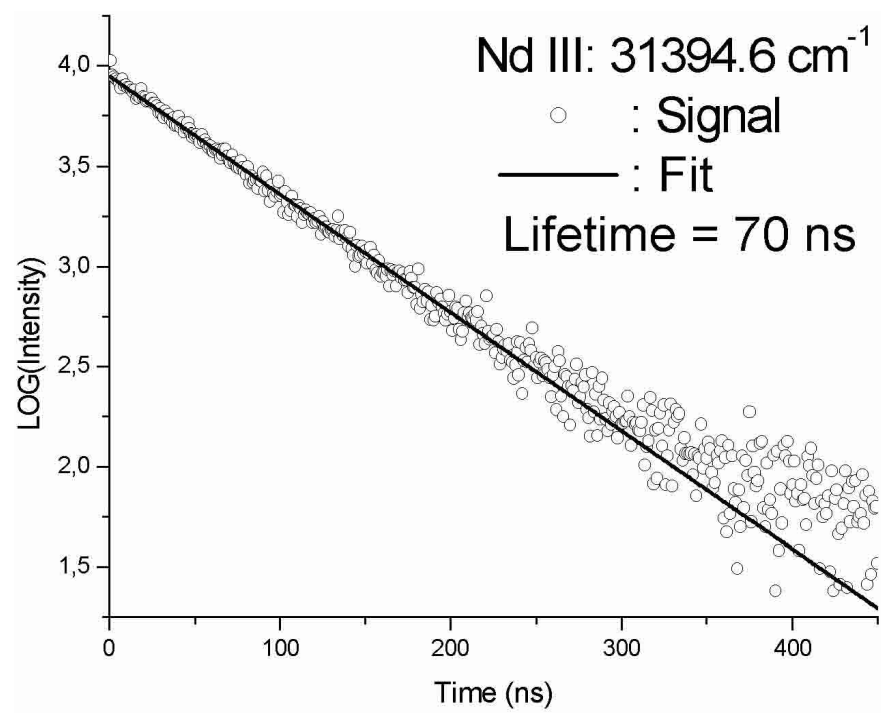

Fig. 2. A typical experimental time-resolved fluorescence signal from the level at $31394.6 \mathrm{~cm}^{-1}$ of Nd III. The lifetime deduced from the fit was $70 \mathrm{~ns}$. The departure from the straight line for values larger than $300 \mathrm{~ns}$ is due to the increasing importance of noise.

flight-out-of-view effects are carefully avoided by enlarging the slit of the monochromator and choosing a suitable delay time.

As a further refinement, a magnetic field of about 60 Gauss was added in the plasma zone by a pair of Helmholtz coils in order to eliminate possible Zeeman quantum beat effects but also to weaken the background associated with the ablation laser and the plasma recombination processes.

In order to obtain a sufficiently high signal-to-noise ratio, a decay curve was obtained by averaging fluorescence photons from more than 2000 pulses. A typical decay curve is shown in Fig. 2.

\section{Theoretical approach}

It has been shown in many previous papers (see e.g. the references quoted at the end of Sect. 1) that, for heavy ions such as singly or doubly ionized REE, the consideration of both intravalence and core-valence correlation is essential for atomic structure calculations. The detailed consideration of configuration interaction effects in complex configurations with an open $f$ shell severely limits the choice of a theoretical method for performing the calculations for the $4 \mathrm{f}^{4}-4 \mathrm{f}^{3} 5 \mathrm{~d}$ transitions in Nd III. In particular, the use of a fully ab initio relativistic approach like the MCDF method must be ruled out in view of the huge number of relativistic states involved and in view of the unrealistic amount of computer time needed for the calculations. In fact, up to now, the various attempts to use the MCDF method for complex situations such as the one considered here have failed. A versatile alternative, which has appeared usable and leads to accurate results (i.e. generally in agreement within a few percent with experimental data obtained with laser techniques) in many different situations, is the relativistic Hartree-Fock technique (HFR) as described and coded by Cowan (1981) but modified for the inclusion of core-polarization effects (see e.g. Palmeri et al. 2000). The lack of convergence problems and the reasonable computer time needed has led us to adopt this approach here.

Detailed comparisons between theoretical calculations and experiment in Er III (Biémont et al. 2001a) and Tm III (Li et al. 2001) have emphasized the fact that the $4 \mathrm{f}-5 \mathrm{~d}$ transitions deserve special attention in lanthanides in relation to the fact that $4 \mathrm{f}$ electrons are deeply imbedded inside the $5 \mathrm{~s}^{2} 5 \mathrm{p}^{6}$ closed shells of the xenon core. A difficulty arises because the analytical core-polarization and core-penetration corrections to the dipole operator are no longer valid (see e.g. Quinet et al. 1999). A possible solution is to apply an empirical scaling factor to the uncorrected $<4 \mathrm{f}|\mathrm{r}| 5 \mathrm{~d}>$ radial matrix element. Although this semi-empirical approach could be useful, it was not followed in the present work. Instead, we have preferred to introduce in the model explicit interactions between the configurations of interest (i.e. $4 \mathrm{f}^{4}$ and $4 \mathrm{f}^{3} 5 \mathrm{~d}$ ) and some higher configurations with three, two or one electron(s) in the $4 \mathrm{f}$ subshell. More precisely, configuration interaction was explicitly retained among the configurations $4 \mathrm{f}^{4}+$ $4 \mathrm{f}^{3} 6 \mathrm{p}+4 \mathrm{f}^{3} 5 \mathrm{f}+4 \mathrm{f}^{3} 6 \mathrm{f}+4 \mathrm{f}^{2} 5 \mathrm{~d}^{2}+4 \mathrm{f}^{2} 6 \mathrm{~s}^{2}+4 \mathrm{f}^{2} 6 \mathrm{p}^{2}+$ $4 \mathrm{f}^{2} 5 \mathrm{~d} 6 \mathrm{~s}$ and $4 \mathrm{f}^{3} 5 \mathrm{~d}+4 \mathrm{f}^{3} 6 \mathrm{~d}+4 \mathrm{f}^{3} 6 \mathrm{~s}+4 \mathrm{f}^{2} 5 \mathrm{~d} 6 \mathrm{p}+4 \mathrm{f}^{2} 6 \mathrm{~s} 6 \mathrm{p}$ $+4 \mathrm{f}^{2} \mathrm{~d}^{3}+4 \mathrm{f} 5 \mathrm{~d}^{2} 6 \mathrm{~s}+4 \mathrm{f} 5 \mathrm{~d} 6 \mathrm{~s}^{2}$. These configuration lists extend considerably those considered by Bord (2000) (i.e. $4 f^{4}+4 f^{2} 5 d^{2}+4 f^{2} 5 d 6 s+4 f^{3} 6 p$ and $\left.4 f^{3} 5 d+4 f^{3} 6 s\right)$ and 
Table 2. Calculated and experimental lifetimes (in ns) obtained in the present work for selected $4 \mathrm{f}^{3} 5 \mathrm{~d}$ levels in Nd III.

\begin{tabular}{lccc}
\hline$E\left(\mathrm{~cm}^{-1}\right)$ & $J$ & Theory & Experiment \\
\hline 27788.2 & 3 & 110 & $110 \pm 10$ \\
28745.3 & 4 & 108 & $170 \pm 10$ \\
30232.3 & 5 & 108 & $105 \pm 15$ \\
31394.6 & 6 & 81 & $70 \pm 5$ \\
32832.6 & 7 & 98 & $87 \pm 7$ \\
\hline
\end{tabular}

are expected to include the most important effects due to intravalence and core-valence correlation. The core-core correlation could a priori be tested by considering the oneand two-electron excitations from the 5 s and 5 p subshells to $4 \mathrm{f}$ but this was prevented, in the present work, by the computer limitations.

The HFR method was combined with a least-squares optimization routine minimizing the discrepancies between observed and calculated energy levels. More precisely, the average energy $\left(E_{\mathrm{av}}\right)$ and the spin-orbit integral $\left(\zeta_{4 \mathrm{f}}\right)$ were adjusted using the five $4 \mathrm{f}^{4}$ levels within the ${ }^{5} \mathrm{I}$ multiplet. The average energy $\left(E_{\mathrm{av}}\right)$, the Slater parameters $\left(F^{\mathrm{k}}, G^{\mathrm{k}}\right)$ and the spin-orbit integrals $\left(\zeta_{4 \mathrm{f}}, \zeta_{5 \mathrm{~d}}\right)$ were optimized using the twenty-four experimental $4 \mathrm{f}^{3} 5 \mathrm{~d}$ levels taken from the NIST compilation (Martin et al. 1978). The effective interaction operators $(\alpha, \beta$ and $\gamma)$ could not be included in the fitting procedure of $4 \mathrm{f}^{3} 5 \mathrm{~d}$ levels because of the lack of experimental level values in this configuration. The radial parameters adopted in the present calculations are reported in Table 3 . For the other configurations (for which no experimental levels are available), a scaling factor of 0.85 was applied to the $F^{\mathrm{k}}, G^{\mathrm{k}}$ and $R^{\mathrm{k}}$ integrals in order to partly take into account interaction with distant configurations not included explicitly in the physical model, according to a well-established procedure. In addition, the average energies were adjusted to reproduce the energies predicted by Brewer (1971) for the lowest level of each configuration, i.e. $4 \mathrm{f}^{3} 6 \mathrm{p}{ }^{5} \mathrm{~K}_{5}$ $\left(E=61000 \pm 2000 \mathrm{~cm}^{-1}\right), 4 \mathrm{f}^{2} 5 \mathrm{~d}^{2}{ }^{5} \mathrm{~L}_{6}(E=72000 \pm$ $\left.5000 \mathrm{~cm}^{-1}\right), 4 \mathrm{f}^{2} 5 \mathrm{~d} 6 \mathrm{~s}{ }^{5} \mathrm{~K}_{5}\left(E=96000 \pm 5000 \mathrm{~cm}^{-1}\right)$, $4 \mathrm{f}^{2} 6 \mathrm{~s}^{2}{ }^{3} \mathrm{H}_{4}\left(E=122000 \pm 7000 \mathrm{~cm}^{-1}\right), 4 \mathrm{f}^{2} 6 \mathrm{p}^{2}{ }^{5} \mathrm{I}_{4}(E=$ $\left.186000 \pm 9000 \mathrm{~cm}^{-1}\right), 4 \mathrm{f}^{3} 6 \mathrm{~s}^{5} \mathrm{I}_{4}^{\circ}\left(E=30500 \pm 2000 \mathrm{~cm}^{-1}\right)$, $4 \mathrm{f}^{2} 5 \mathrm{~d} 6 \mathrm{p}{ }^{5} \mathrm{~L}_{6}^{\circ}\left(E=126000 \pm 4000 \mathrm{~cm}^{-1}\right)$ and $4 \mathrm{f}^{2} 6 \mathrm{~s} 6 \mathrm{p}{ }^{5} \mathrm{I}_{4}^{\circ}$ $\left(E=154000 \pm 7000 \mathrm{~cm}^{-1}\right)$.

The calculated energy levels are compared with experiments, when available, in Table 4 for odd-parity levels below $33000 \mathrm{~cm}^{-1}$. In view of the very good agreement between theory and experiment (the mean deviation is $0.23 \%$ ), the new calculated levels are expected to be reliable to a few tens of percent and, consequently, can be used to support future analysis and identification work. We report also in Table 4 the calculated Landé $g$-factors. They agree well with the values calculated by Bord (2000): $\Delta=0.014 \pm 0.023$ for 34 odd levels, the largest discrepancies appearing, as expected, for the strongly perturbed levels: $E=27569.8,27788.2$ and $29397.3 \mathrm{~cm}^{-1}$. There are no other $g$-factors available for comparison.
Table 3. Radial parameters adopted in the present calculations for the $4 \mathrm{f}^{4}$ and $4 \mathrm{f}^{3} 5 \mathrm{~d}$ configurations in Nd III.

\begin{tabular}{llrrc}
\hline Config. & Parameter & $\begin{array}{r}\text { Ab initio } \\
\left(\mathrm{cm}^{-1}\right)\end{array}$ & $\begin{array}{r}\text { Fit } \\
\left(\mathrm{cm}^{-1}\right)\end{array}$ & Fit/ab initio \\
\hline $4 \mathrm{f}^{4}$ & $E_{\mathrm{av}}$ & & 35392 & \\
& $\mathrm{~F}^{2}(4 \mathrm{f}, 4 \mathrm{f})$ & 92635 & 78740 & $0.850^{*}$ \\
& $\mathrm{~F}^{4}(4 \mathrm{f}, 4 \mathrm{f})$ & 57680 & 49028 & $0.850^{*}$ \\
& $\mathrm{~F}^{6}(4 \mathrm{f}, 4 \mathrm{f})$ & 41373 & 35167 & $0.850^{*}$ \\
& $\zeta_{4 \mathrm{f}}$ & 849 & 780 & 0.919 \\
$4 \mathrm{f}^{3} 5 \mathrm{~d}$ & & & \\
& $E_{\mathrm{av}}$ & & 43230 & \\
& $\mathrm{~F}^{2}(4 \mathrm{f}, 4 \mathrm{f})$ & 101378 & 64485 & 0.636 \\
& $\mathrm{~F}^{4}(4 \mathrm{f}, 4 \mathrm{f})$ & 63552 & 65286 & 1.027 \\
& $\mathrm{~F}^{6}(4 \mathrm{f}, 4 \mathrm{f})$ & 54705 & 28855 & 0.631 \\
& $\zeta_{4 \mathrm{f}}$ & 947 & 898 & 0.948 \\
& $\zeta_{5 \mathrm{~d}}$ & 831 & 755 & 0.909 \\
& $\mathrm{~F}^{2}(4 \mathrm{f}, 5 \mathrm{~d})$ & 26408 & 16541 & 0.626 \\
& $\mathrm{~F}^{4}(4 \mathrm{f}, 5 \mathrm{~d})$ & 13021 & 8809 & 0.677 \\
& $\mathrm{G}^{1}(4 \mathrm{f}, 5 \mathrm{~d})$ & 12823 & 9161 & 0.714 \\
& $\mathrm{G}^{3}(4 \mathrm{f}, 5 \mathrm{~d})$ & 10202 & 4251 & 0.417 \\
& $\mathrm{G}^{5}(4 \mathrm{f}, 5 \mathrm{~d})$ & 7735 & 9805 & 1.268 \\
\hline
\end{tabular}

* Fixed value.

Experimental and theoretical radiative lifetimes obtained in the present work are compared in Table 2. As seen from the table, the agreement between both sets of values is very good, the average percentage error being $7.7 \%$ without the $28745.3 \mathrm{~cm}^{-1}$ level, and $13.5 \%$ with it. For this level, the calculated lifetime $\left(\tau_{\text {calc }}=108 \mathrm{~ns}\right)$ is a factor of 1.6 shorter than the measurement $\left(\tau_{\exp }=\right.$ $170 \pm 10 \mathrm{~ns})$. There is no clear reason for this discrepancy. It could possibly be attributed to a wrong intermediate coupling representation of the calculated eigenvector, the level appearing strongly mixed (see Table 4). However, some of the levels, for which the agreement with experiment is good, also appear strongly mixed.

The computed oscillator strengths $(\log g f)$ and transition probabilities $(g A)$ are listed in Table 5 alongside the lower and upper experimental energy levels of the transitions and the air wavelengths in nm. These wavelengths were derived from the Nd III experimental levels compiled by Martin et al. (1978). Only transitions with $\log g f>-4.0$ are reported in Table 5 . We give in Table 5 both the HFR $g A$-values calculated as above described and also the weighted transition probabilities normalized according to the new laser lifetime measurements. The comparison between our $g f$-values and the results published by Bord (2000) is illustrated in Fig. 3. As seen from this figure, the general agreement between both sets of values is very gratifying (as expected because two different versions of the same code were used). Exceptions occur for the lines at 353.7622, 370.1320, 496.2798 and $545.3159 \mathrm{~nm}$. For these transitions, our $\log g f$-values are equal to -2.65 , $-1.05,-3.45$ and -3.85 , respectively while the Bord's results are $-3.29,-2.29,-6.08$ and -7.52 . However, for the latter two lines (496.2798 and $545.3159 \mathrm{~nm}$ ), the discrepancies are obviously related to the fact that the 
Table 4. Low-lying odd energy levels $\left(E<33000 \mathrm{~cm}^{-1}\right)$ of Nd III.

\begin{tabular}{|c|c|c|c|c|c|c|}
\hline Config. & $\mathrm{J}$ & $\begin{array}{c}E_{\exp }^{\mathrm{a}} \\
\left(\mathrm{cm}^{-1}\right)\end{array}$ & $\begin{array}{c}E_{\text {calc }}^{\mathrm{b}} \\
\left(\mathrm{cm}^{-1}\right)\end{array}$ & $\begin{array}{r}\Delta E^{\mathrm{c}} \\
\left(\mathrm{cm}^{-1}\right)\end{array}$ & $g_{\text {calc }}^{\mathrm{d}}$ & Eigenvector composition $^{\mathrm{e}}$ \\
\hline $4 f^{3} 5 d$ & 6 & & 15290 & & 0.724 & $93 \%\left({ }^{4} \mathrm{I}^{\circ}\right) 5 \mathrm{~d}{ }^{5} \mathrm{~L}^{\circ}$ \\
\hline $4 \mathrm{f}^{3} 5 \mathrm{~d}$ & 5 & 15262.2 & 15322 & -60 & 0.683 & $90 \%\left({ }^{4} \mathrm{I}^{\circ}\right) 5 \mathrm{~d}{ }^{5} \mathrm{~K}^{\circ}+6 \%\left({ }^{4} \mathrm{I}^{\circ}\right) 5 \mathrm{~d}{ }^{3} \mathrm{I}^{\circ}$ \\
\hline $4 \mathrm{f}^{3} 5 \mathrm{~d}$ & 6 & 16938.1 & 16944 & -6 & 0.910 & $95 \%\left({ }^{4} \mathrm{I}^{\circ}\right) 5 \mathrm{~d} \quad{ }^{5} \mathrm{~K}^{\circ}$ \\
\hline $4 f^{3} 5 d$ & 7 & & 17077 & & 0.915 & $96 \%\left({ }^{4} \mathrm{I}^{\circ}\right) 5 \mathrm{~d}{ }^{5} \mathrm{~L}^{\circ}$ \\
\hline $4 \mathrm{f}^{3} 5 \mathrm{~d}$ & 7 & 18656.3 & 18628 & 28 & 1.053 & $97 \%\left({ }^{4} \mathrm{I}^{\circ}\right) 5 \mathrm{~d}{ }^{5} \mathrm{~K}^{\circ}$ \\
\hline $4 f^{3} 5 d$ & 4 & 18883.7 & 18819 & 65 & 0.625 & $88 \%\left({ }^{4} \mathrm{I}^{\circ}\right) 5 \mathrm{~d} \quad{ }^{5} \mathrm{I}^{\circ}+9 \%\left({ }^{4} \mathrm{I}^{\circ}\right) 5 \mathrm{~d}{ }^{3} \mathrm{H}^{\circ}$ \\
\hline $4 f^{3} 5 d$ & 8 & & 18980 & & 1.043 & $98 \%\left({ }^{4} \mathrm{I}^{\circ}\right) 5 \mathrm{~d}{ }^{5} \mathrm{~L}^{\circ}$ \\
\hline $4 f^{3} 5 d$ & 3 & 19211.0 & 19271 & -60 & 0.547 & $77 \%\left({ }^{4} \mathrm{I}^{\circ}\right) 5 \mathrm{~d}{ }^{5} \mathrm{H}^{\circ}+10 \%\left({ }^{4} \mathrm{I}^{\circ}\right) 5 \mathrm{~d}{ }^{3} \mathrm{G}^{\circ}+7 \%\left({ }^{4} \mathrm{~F}^{\circ}\right) 5 \mathrm{~d}{ }^{5} \mathrm{H}^{\circ}$ \\
\hline $4 f^{3} 5 d$ & 5 & & 19740 & & 0.887 & $40 \%\left({ }^{4} \mathrm{I}^{\circ}\right) 5 \mathrm{~d}{ }^{3} \mathrm{I}^{\circ}+36 \%\left({ }^{4} \mathrm{I}^{\circ}\right) 5 \mathrm{~d}{ }^{5} \mathrm{I}^{\circ}+6 \%\left({ }^{4} \mathrm{I}^{\circ}\right) 5 \mathrm{~d}{ }^{5} \mathrm{H}^{\circ}$ \\
\hline $4 f^{3} 5 d$ & 4 & 20144.3 & 20082 & 62 & 0.871 & $64 \%\left({ }^{4} \mathrm{I}^{\circ}\right) 5 \mathrm{~d}{ }^{5} \mathrm{H}^{\circ}+18 \%\left({ }^{4} \mathrm{I}^{\circ}\right) 5 \mathrm{~d}{ }^{3} \mathrm{H}^{\circ}$ \\
\hline $4 \mathrm{f}^{3} 5 \mathrm{~d}$ & 2 & & 20218 & & 0.343 & $94 \%\left({ }^{4} \mathrm{I}^{\circ}\right) 5 \mathrm{~d}{ }^{5} \mathrm{G}^{\circ}$ \\
\hline $4 \mathrm{f}^{3} 5 \mathrm{~d}$ & 8 & 20410.9 & 20373 & 38 & 1.149 & $96 \%\left({ }^{4} \mathrm{I}^{\circ}\right) 5 \mathrm{~d} \quad{ }^{5} \mathrm{~K}^{\circ}$ \\
\hline $4 \mathrm{f}^{3} 5 \mathrm{~d}$ & 5 & 20388.9 & 20388 & 1 & 0.936 & $56 \%\left({ }^{4} \mathrm{I}^{\circ}\right) 5 \mathrm{~d}{ }^{5} \mathrm{I}^{\circ}+15 \%\left({ }^{4} \mathrm{I}^{\circ}\right) 5 \mathrm{~d}{ }^{3} \mathrm{H}^{\circ}+12 \%\left({ }^{4} \mathrm{I}^{\circ}\right) 5 \mathrm{~d}{ }^{3} \mathrm{I}^{\circ}$ \\
\hline $4 \mathrm{f}^{3} 5 \mathrm{~d}$ & 3 & & 20560 & & 0.870 & $77 \%\left({ }^{4} \mathrm{I}^{\circ}\right) 5 \mathrm{~d}{ }^{5} \mathrm{G}^{\circ}+10 \%\left({ }^{4} \mathrm{I}^{\circ}\right) 5 \mathrm{~d}{ }^{3} \mathrm{G}^{\circ}+7 \%\left({ }^{4} \mathrm{I}^{\circ}\right) 5 \mathrm{~d}{ }^{5} \mathrm{H}^{\circ}$ \\
\hline $4 \mathrm{f}^{3} 5 \mathrm{~d}$ & 6 & & 20822 & & 1.029 & $33 \%\left({ }^{4} \mathrm{I}^{\circ}\right) 5 \mathrm{~d}{ }^{5} \mathrm{I}^{\circ}+32 \%\left({ }^{4} \mathrm{I}^{\circ}\right) 5 \mathrm{~d}{ }^{3} \mathrm{I}^{\circ}+14 \%\left({ }^{4} \mathrm{I}^{\circ}\right) 5 \mathrm{~d}{ }^{3} \mathrm{~K}^{\circ}$ \\
\hline $4 f^{3} 5 d$ & 9 & & 20975 & & 1.133 & $99 \%\left({ }^{4} \mathrm{I}^{\circ}\right) 5 \mathrm{~d} \quad{ }^{5} \mathrm{~L}^{\circ}$ \\
\hline $4 \mathrm{f}^{3} 5 \mathrm{~d}$ & 4 & & 21183 & & 1.034 & $51 \%\left({ }^{4} \mathrm{I}^{\circ}\right) 5 \mathrm{~d}{ }^{5} \mathrm{G}^{\circ}+15 \%\left({ }^{4} \mathrm{I}^{\circ}\right) 5 \mathrm{~d}{ }^{3} \mathrm{G}^{\circ}+13 \%\left({ }^{4} \mathrm{I}^{\circ}\right) 5 \mathrm{~d}{ }^{3} \mathrm{H}^{\circ}$ \\
\hline $4 \mathrm{f}^{3} 5 \mathrm{~d}$ & 5 & 21886.8 & 21878 & 9 & 1.021 & $61 \%\left({ }^{4} \mathrm{I}^{\circ}\right) 5 \mathrm{~d}{ }^{5} \mathrm{H}^{\circ}+22 \%\left({ }^{4} \mathrm{I}^{\circ}\right) 5 \mathrm{~d}{ }^{3} \mathrm{I}^{\circ}$ \\
\hline $4 f^{3} 5 d$ & 6 & 22047.8 & 22048 & 0 & 1.088 & $60 \%\left({ }^{4} \mathrm{I}^{\circ}\right) 5 \mathrm{~d}{ }^{5} \mathrm{I}^{\circ}+12 \%\left({ }^{4} \mathrm{I}^{\circ}\right) 5 \mathrm{~d}{ }^{5} \mathrm{H}^{\circ}+10 \%\left({ }^{4} \mathrm{I}^{\circ}\right) 5 \mathrm{~d}{ }^{3} \mathrm{I}^{\circ}$ \\
\hline $4 f^{3} 5 d$ & 9 & 22197.0 & 22171 & 26 & 1.217 & $95 \%\left({ }^{4} \mathrm{I}^{\circ}\right) 5 \mathrm{~d}{ }^{5} \mathrm{~K}^{\circ}$ \\
\hline $4 f^{3} 5 d$ & 4 & & 22438 & & 0.934 & $39 \%\left({ }^{4} \mathrm{I}^{\circ}\right) 5 \mathrm{~d}{ }^{3} \mathrm{H}^{\circ}+34 \%\left({ }^{4} \mathrm{I}^{\circ}\right) 5 \mathrm{~d}{ }^{5} \mathrm{G}^{\circ}+11 \%\left({ }^{4} \mathrm{I}^{\circ}\right) 5 \mathrm{~d}{ }^{5} \mathrm{H}^{\circ}$ \\
\hline $4 \mathrm{f}^{3} 5 \mathrm{~d}$ & 7 & 22702.9 & 22665 & 37 & 1.151 & $61 \%\left({ }^{4} \mathrm{I}^{\circ}\right) 5 \mathrm{~d}{ }^{5} \mathrm{I}^{\circ}+17 \%\left({ }^{4} \mathrm{I}^{\circ}\right) 5 \mathrm{~d}{ }^{3} \mathrm{I}^{\circ}+13 \%\left({ }^{4} \mathrm{I}^{\circ}\right) 5 \mathrm{~d}{ }^{3} \mathrm{~K}^{\circ}$ \\
\hline $4 \mathrm{f}^{3} 5 \mathrm{~d}$ & 5 & & 22787 & & 1.215 & $71 \%\left({ }^{4} \mathrm{I}^{\circ}\right) 5 \mathrm{~d}{ }^{5} \mathrm{G}^{\circ}+7 \%\left({ }^{4} \mathrm{I}^{\circ}\right) 5 \mathrm{~d}{ }^{3} \mathrm{G}^{\circ}+7 \%\left({ }^{4} \mathrm{I}^{\circ}\right) 5 \mathrm{~d}{ }^{5} \mathrm{H}^{\circ}$ \\
\hline $4 \mathrm{f}^{3} 5 \mathrm{~d}$ & 6 & & 22918 & & 0.991 & $47 \%\left({ }^{4} \mathrm{I}^{\circ}\right) 5 \mathrm{~d}{ }^{3} \mathrm{~K}^{\circ}+28 \%\left({ }^{4} \mathrm{I}^{\circ}\right) 5 \mathrm{~d}{ }^{5} \mathrm{H}^{\circ}+6 \%\left({ }^{2} \mathrm{H}^{\circ}\right) 5 \mathrm{~d}{ }^{3} \mathrm{~K}^{\circ}$ \\
\hline $4 f^{3} 5 d$ & 10 & & 23041 & & 1.199 & $99 \%\left({ }^{4} \mathrm{I}^{\circ}\right) 5 \mathrm{~d}{ }^{5} \mathrm{~L}^{\circ}$ \\
\hline $4 f^{3} 5 d$ & 7 & & 23694 & & 0.891 & $82 \%\left({ }^{4} \mathrm{I}^{\circ}\right) 5 \mathrm{~d}{ }^{3} \mathrm{~L}^{\circ}+7 \%\left({ }^{2} \mathrm{~K}^{\circ}\right) 5 \mathrm{~d}{ }^{3} \mathrm{~L}^{\circ}$ \\
\hline $4 f^{3} 5 d$ & 6 & 23819.3 & 23953 & -134 & 1.075 & $38 \%\left({ }^{4} \mathrm{I}^{\circ}\right) 5 \mathrm{~d}{ }^{5} \mathrm{H}^{\circ}+34 \%\left({ }^{4} \mathrm{I}^{\circ}\right) 5 \mathrm{~d}{ }^{3} \mathrm{I}^{\circ}+13 \%\left({ }^{4} \mathrm{I}^{\circ}\right) 5 \mathrm{~d}{ }^{3} \mathrm{~K}^{\circ}$ \\
\hline $4 f^{3} 5 d$ & 3 & & 24034 & & 0.771 & $72 \%\left({ }^{4} \mathrm{I}^{\circ}\right) 5 \mathrm{~d}{ }^{3} \mathrm{G}^{\circ}+16 \%\left({ }^{4} \mathrm{I}^{\circ}\right) 5 \mathrm{~d}{ }^{5} \mathrm{G}^{\circ}+6 \%\left({ }^{4} \mathrm{I}^{\circ}\right) 5 \mathrm{~d}{ }^{5} \mathrm{H}^{\circ}$ \\
\hline $4 f^{3} 5 d$ & 7 & 24003.2 & 24093 & -90 & 1.196 & $33 \%\left({ }^{4} \mathrm{I}^{\circ}\right) 5 \mathrm{~d}{ }^{5} \mathrm{H}^{\circ}+30 \%\left({ }^{4} \mathrm{I}^{\circ}\right) 5 \mathrm{~d}{ }^{5} \mathrm{I}^{\circ}+23 \%\left({ }^{4} \mathrm{I}^{\circ}\right) 5 \mathrm{~d}{ }^{3} \mathrm{I}^{\circ}$ \\
\hline $4 f^{3} 5 d$ & 6 & & 24481 & & 1.295 & $79 \%\left({ }^{4} \mathrm{I}^{\circ}\right) 5 \mathrm{~d}{ }^{5} \mathrm{G}^{\circ}+7 \%\left({ }^{4} \mathrm{I}^{\circ}\right) 5 \mathrm{~d}{ }^{3} \mathrm{H}^{\circ}$ \\
\hline $4 f^{3} 5 d$ & 8 & 24686.4 & 24605 & 81 & 1.230 & $84 \%\left({ }^{4} \mathrm{I}^{\circ}\right) 5 \mathrm{~d}{ }^{5} \mathrm{I}^{\circ}+12 \%\left({ }^{4} \mathrm{I}^{\circ}\right) 5 \mathrm{~d}{ }^{3} \mathrm{~K}^{\circ}$ \\
\hline $4 f^{3} 5 d$ & 5 & & 24653 & & 1.067 & $51 \%\left({ }^{4} \mathrm{I}^{\circ}\right) 5 \mathrm{~d}{ }^{3} \mathrm{H}^{\circ}+18 \%\left({ }^{4} \mathrm{I}^{\circ}\right) 5 \mathrm{~d}{ }^{5} \mathrm{G}^{\circ}+7 \%\left({ }^{4} \mathrm{I}^{\circ}\right) 5 \mathrm{~d}{ }^{5} \mathrm{H}^{\circ}$ \\
\hline $4 \mathrm{f}^{3} 5 \mathrm{~d}$ & 7 & & 25039 & & 1.123 & $45 \%\left({ }^{4} \mathrm{I}^{\circ}\right) 5 \mathrm{~d}{ }^{3} \mathrm{~K}^{\circ}+31 \%\left({ }^{4} \mathrm{I}^{\circ}\right) 5 \mathrm{~d}{ }^{5} \mathrm{H}^{\circ}+8 \%\left({ }^{4} \mathrm{I}^{\circ}\right) 5 \mathrm{~d}{ }^{5} \mathrm{I}^{\circ}$ \\
\hline $4 f^{3} 5 d$ & 0 & & 25883 & & & $33 \%\left({ }^{4} \mathrm{~F}^{\circ}\right) 5 \mathrm{~d}{ }^{5} \mathrm{D}^{\circ}+25 \%\left({ }^{4} \mathrm{~S}^{\circ}\right) 5 \mathrm{~d}{ }^{5} \mathrm{D}^{\circ}+20 \%\left({ }^{4} \mathrm{~F}^{\circ}\right) 5 \mathrm{~d}{ }^{3} \mathrm{P}^{\circ}$ \\
\hline $4 f^{3} 5 d$ & 8 & & 25894 & & 1.020 & $84 \%\left({ }^{4} \mathrm{I}^{\circ}\right) 5 \mathrm{~d}{ }^{3} \mathrm{~L}^{\circ}+8 \%\left({ }^{2} \mathrm{~K}^{\circ}\right) 5 \mathrm{~d}{ }^{3} \mathrm{~L}^{\circ}$ \\
\hline $4 f^{3} 5 d$ & 7 & & 26120 & & 1.139 & $43 \%\left({ }^{4} \mathrm{I}^{\circ}\right) 5 \mathrm{~d}{ }^{3} \mathrm{I}^{\circ}+22 \%\left({ }^{4} \mathrm{I}^{\circ}\right) 5 \mathrm{~d}{ }^{3} \mathrm{~K}^{\circ}+20 \%\left({ }^{4} \mathrm{I}^{\circ}\right) 5 \mathrm{~d}{ }^{5} \mathrm{H}^{\circ}$ \\
\hline $4 \mathrm{f}^{3} 5 \mathrm{~d}$ & 6 & 26503.2 & 26564 & -61 & 1.178 & $48 \%\left({ }^{4} \mathrm{I}^{\circ}\right) 5 \mathrm{~d}{ }^{3} \mathrm{H}^{\circ}+14 \%\left({ }^{4} \mathrm{I}^{\circ}\right) 5 \mathrm{~d}{ }^{5} \mathrm{G}^{\circ}+8 \%\left({ }^{4} \mathrm{G}^{\circ}\right) 5 \mathrm{~d}{ }^{3} \mathrm{H}^{\circ}$ \\
\hline $4 f^{3} 5 d$ & 4 & & 26608 & & 1.049 & $79 \%\left({ }^{4} \mathrm{I}^{\circ}\right) 5 \mathrm{~d}{ }^{3} \mathrm{G}^{\circ}+10 \%\left({ }^{4} \mathrm{I}^{\circ}\right) 5 \mathrm{~d}{ }^{5} \mathrm{G}^{\circ}$ \\
\hline $4 f^{3} 5 d$ & 1 & & 26787 & & 1.489 & $42 \%\left({ }^{4} \mathrm{~F}^{\circ}\right) 5 \mathrm{~d}{ }^{5} \mathrm{D}^{\circ}+39 \%\left({ }^{4} \mathrm{~S}^{\circ}\right) 5 \mathrm{~d}{ }^{5} \mathrm{D}^{\circ}+7 \%\left({ }^{4} \mathrm{~F}^{\circ}\right) 5 \mathrm{~d}{ }^{3} \mathrm{P}^{\circ}$ \\
\hline $4 f^{3} 5 d$ & 2 & & 27295 & & 1.446 & $41 \%\left({ }^{4} \mathrm{~S}^{\circ}\right) 5 \mathrm{~d}{ }^{5} \mathrm{D}^{\circ}+40 \%\left({ }^{4} \mathrm{~F}^{\circ}\right) 5 \mathrm{~d}{ }^{5} \mathrm{D}^{\circ}$ \\
\hline $4 f^{3} 5 d$ & 8 & 27391.4 & 27300 & 91 & 1.138 & $71 \%\left({ }^{4} \mathrm{I}^{\circ}\right) 5 \mathrm{~d}{ }^{3} \mathrm{~K}^{\circ}+12 \%\left({ }^{4} \mathrm{I}^{\circ}\right) 5 \mathrm{~d}{ }^{5} \mathrm{I}^{\circ}+10 \%\left({ }^{2} \mathrm{H}^{\circ}\right) 5 \mathrm{~d}{ }^{3} \mathrm{~K}^{\circ}$ \\
\hline $4 f^{3} 5 d$ & 3 & 27569.8 & 27433 & 137 & 1.085 & $26 \%\left({ }^{4} \mathrm{~F}^{\circ}\right) 5 \mathrm{~d}{ }^{5} \mathrm{H}^{\circ}+21 \%\left({ }^{4} \mathrm{~S}^{\circ}\right) 5 \mathrm{~d}{ }^{5} \mathrm{D}^{\circ}+19 \%\left({ }^{4} \mathrm{~F}^{\circ}\right) 5 \mathrm{~d}{ }^{5} \mathrm{D}^{\circ}$ \\
\hline $4 f^{3} 5 d$ & 2 & & 27525 & & 0.395 & $83 \%\left({ }^{4} \mathrm{~F}^{\circ}\right) 5 \mathrm{~d} \quad{ }^{5} \mathrm{G}^{\circ}$ \\
\hline $4 f^{3} 5 d$ & 3 & 27788.2 & 27749 & 39 & 0.873 & $53 \%\left({ }^{4} \mathrm{~F}^{\circ}\right) 5 \mathrm{~d}{ }^{5} \mathrm{H}^{\circ}+15 \%\left({ }^{4} \mathrm{~S}^{\circ}\right) 5 \mathrm{~d}{ }^{5} \mathrm{D}^{\circ}+14 \%\left({ }^{4} \mathrm{~F}^{\circ}\right) 5 \mathrm{~d}{ }^{5} \mathrm{D}^{\circ}$ \\
\hline $4 f^{3} 5 d$ & 9 & & 28109 & & 1.114 & $83 \%\left({ }^{4} \mathrm{I}^{\circ}\right) 5 \mathrm{~d}{ }^{3} \mathrm{~L}^{\circ}+7 \%\left({ }^{2} \mathrm{~K}^{\circ}\right) 5 \mathrm{~d}{ }^{3} \mathrm{~L}^{\circ}$ \\
\hline $4 f^{3} 5 d$ & 3 & & 28532 & & 0.921 & $85 \%\left({ }^{4} \mathrm{~F}^{\circ}\right) 5 \mathrm{~d}{ }^{5} \mathrm{G}^{\circ}$ \\
\hline $4 f^{3} 5 d$ & 4 & & 28657 & & 1.249 & $29 \%\left({ }^{4} \mathrm{~F}^{\circ}\right) 5 \mathrm{~d}{ }^{5} \mathrm{H}^{\circ}+25 \%\left({ }^{4} \mathrm{~S}^{\circ}\right) 5 \mathrm{~d}{ }^{5} \mathrm{D}^{\circ}+22 \%\left({ }^{4} \mathrm{~F}^{\circ}\right) 5 \mathrm{~d}{ }^{5} \mathrm{D}^{\circ}$ \\
\hline $4 \mathrm{f}^{3} 5 \mathrm{~d}$ & 4 & 28745.3 & 28839 & -94 & 1.102 & $56 \%\left({ }^{4} \mathrm{~F}^{\circ}\right) 5 \mathrm{~d}{ }^{5} \mathrm{H}^{\circ}+16 \%\left({ }^{4} \mathrm{~S}^{\circ}\right) 5 \mathrm{~d}{ }^{5} \mathrm{D}^{\circ}+14 \%\left({ }^{4} \mathrm{~F}^{\circ}\right) 5 \mathrm{~d}{ }^{5} \mathrm{D}^{\circ}$ \\
\hline $4 f^{3} 5 d$ & 5 & & 29204 & & 1.194 & $84 \%\left({ }^{4} \mathrm{I}^{\circ}\right) 5 \mathrm{~d}{ }^{3} \mathrm{G}^{\circ}$ \\
\hline $4 f^{3} 5 d$ & 0 & & 29361 & & & $43 \%\left({ }^{4} \mathrm{~F}^{\circ}\right) 5 \mathrm{~d}{ }^{3} \mathrm{P}^{\circ}+29 \%\left({ }^{4} \mathrm{~S}^{\circ}\right) 5 \mathrm{~d}{ }^{5} \mathrm{D}^{\circ}+9 \%\left({ }^{2} \mathrm{D}^{\circ}\right) 5 \mathrm{~d}{ }^{1} \mathrm{~S}^{\circ}$ \\
\hline $4 f^{3} 5 d$ & 4 & & 29440 & & 1.132 & $78 \%\left({ }^{4} \mathrm{~F}^{\circ}\right) 5 \mathrm{~d}{ }^{5} \mathrm{G}^{\circ}$ \\
\hline $4 f^{3} 5 d$ & 5 & 29397.3 & 29519 & -122 & 1.036 & $31 \%\left({ }^{4} \mathrm{~F}^{\circ}\right) 5 \mathrm{~d}{ }^{5} \mathrm{H}^{\circ}+14 \%\left({ }^{2} \mathrm{H}^{\circ}\right) 5 \mathrm{~d}{ }^{3} \mathrm{I}^{\circ}+12 \%\left({ }^{4} \mathrm{~F}^{\circ}\right) 5 \mathrm{~d}{ }^{5} \mathrm{G}^{\circ}$ \\
\hline $4 \mathrm{f}^{3} 5 \mathrm{~d}$ & 6 & & 29626 & & 1.018 & $23 \%\left({ }^{2} \mathrm{H}^{\circ}\right) 5 \mathrm{~d}{ }^{3} \mathrm{~K}^{\circ}+12 \%\left({ }^{4} \mathrm{I}^{\circ}\right) 5 \mathrm{~d}{ }^{3} \mathrm{~K}^{\circ}+9 \%\left({ }^{2} \mathrm{H}^{\circ}\right) 5 \mathrm{~d}{ }^{1} \mathrm{I}^{\circ}$ \\
\hline $4 \mathrm{f}^{3} 5 \mathrm{~d}$ & 1 & & 29840 & & 1.273 & $29 \%\left({ }^{4} \mathrm{~F}^{\circ}\right) 5 \mathrm{~d}{ }^{5} \mathrm{P}^{\circ}+24 \%\left({ }^{4} \mathrm{~F}^{\circ}\right) 5 \mathrm{~d}{ }^{3} \mathrm{P}^{\circ}+23 \%\left({ }^{4} \mathrm{~F}^{\circ}\right) 5 \mathrm{~d}{ }^{5} \mathrm{~F}^{\circ}$ \\
\hline $4 \mathrm{f}^{3} 5 \mathrm{~d}$ & 2 & & 29991 & & 1.191 & $20 \%\left({ }^{4} \mathrm{~F}^{\circ}\right) 5 \mathrm{~d}{ }^{3} \mathrm{D}^{\circ}+19 \%\left({ }^{4} \mathrm{~F}^{\circ}\right) 5 \mathrm{~d}{ }^{5} \mathrm{P}^{\circ}+11 \%\left({ }^{4} \mathrm{~F}^{\circ}\right) 5 \mathrm{~d}{ }^{3} \mathrm{~F}^{\circ}$ \\
\hline $4 \mathrm{f}^{3} 5 \mathrm{~d}$ & 5 & 30232.3 & 30198 & 34 & 1.142 & $49 \%\left({ }^{4} \mathrm{~F}^{\circ}\right) 5 \mathrm{~d}{ }^{5} \mathrm{H}^{\circ}+30 \%\left({ }^{4} \mathrm{~F}^{\circ}\right) 5 \mathrm{~d}{ }^{5} \mathrm{G}^{\circ}$ \\
\hline $4 f^{3} 5 d$ & 1 & & 30298 & & 1.057 & $51 \%\left({ }^{4} \mathrm{~F}^{\circ}\right) 5 \mathrm{~d}{ }^{5} \mathrm{~F}^{\circ}+38 \%\left({ }^{4} \mathrm{~F}^{\circ}\right) 5 \mathrm{~d}{ }^{5} \mathrm{P}^{\circ}$ \\
\hline $4 f^{3} 6 s$ & 4 & & 30431 & & 0.611 & $92 \%\left({ }^{4} \mathrm{I}^{\circ}\right) 6 \mathrm{~s}{ }^{5} \mathrm{I}^{\circ}$ \\
\hline
\end{tabular}


Table 4. continued.

\begin{tabular}{|c|c|c|c|c|c|c|}
\hline Config. & $\mathrm{J}$ & $\begin{array}{c}E_{\exp }^{\mathrm{a}} \\
\left(\mathrm{cm}^{-1}\right)\end{array}$ & $\begin{array}{c}E_{\text {calc }}^{\mathrm{b}} \\
\left(\mathrm{cm}^{-1}\right)\end{array}$ & $\begin{array}{c}\Delta E^{\mathrm{c}} \\
\left(\mathrm{cm}^{-1}\right)\end{array}$ & $g_{\text {calc }}^{\mathrm{d}}$ & Eigenvector composition $^{\mathrm{e}}$ \\
\hline $4 f^{3} 5 d$ & 3 & & 30702 & & 1.116 & $13 \%\left({ }^{4} \mathrm{~S}^{\circ}\right) 5 \mathrm{~d}{ }^{5} \mathrm{D}^{\circ}+12 \%\left({ }^{2} \mathrm{H}^{\circ}\right) 5 \mathrm{~d}{ }^{3} \mathrm{G}^{\circ}+9 \%\left({ }^{4} \mathrm{~F}^{\circ}\right) 5 \mathrm{~d}{ }^{3} \mathrm{~F}^{\circ}$ \\
\hline $4 f^{3} 5 d$ & 1 & & 30768 & & 1.420 & $28 \%\left({ }^{4} \mathrm{~F}^{\circ}\right) 5 \mathrm{~d}{ }^{3} \mathrm{P}^{\circ}+25 \%\left({ }^{4} \mathrm{~F}^{\circ}\right) 5 \mathrm{~d}{ }^{5} \mathrm{P}^{\circ}+12 \%\left({ }^{4} \mathrm{~F}^{\circ}\right) 5 \mathrm{~d}{ }^{5} \mathrm{~F}^{\circ}$ \\
\hline $4 f^{3} 5 d$ & 2 & & 30860 & & 1.118 & $75 \%\left({ }^{4} \mathrm{~F}^{\circ}\right) 5 \mathrm{~d}{ }^{5} \mathrm{~F}^{\circ}+14 \%\left({ }^{4} \mathrm{~F}^{\circ}\right) 5 \mathrm{~d}{ }^{5} \mathrm{P}^{\circ}$ \\
\hline $4 \mathrm{f}^{3} 5 \mathrm{~d}$ & 5 & & 30898 & & 1.009 & $30 \%\left({ }^{4} \mathrm{~F}^{\circ}\right) 5 \mathrm{~d}{ }^{5} \mathrm{G}^{\circ}+17 \%\left({ }^{2} \mathrm{H}^{\circ}\right) 5 \mathrm{~d}{ }^{3} \mathrm{I}^{\circ}+16 \%\left({ }^{4} \mathrm{I}^{\circ}\right) 6 \mathrm{~s}{ }^{5} \mathrm{I}^{\circ}$ \\
\hline $4 \mathrm{f}^{3} 5 \mathrm{~d}$ & 4 & & 30918 & & 0.884 & $31 \%\left({ }^{4} \mathrm{~F}^{\circ}\right) 5 \mathrm{~d}{ }^{3} \mathrm{H}^{\circ}+15 \%\left({ }^{2} \mathrm{H}^{\circ}\right) 5 \mathrm{~d}{ }^{3} \mathrm{H}^{\circ}+10 \%\left({ }^{4} \mathrm{~F}^{\circ}\right) 5 \mathrm{~d}{ }^{5} \mathrm{G}^{\circ}$ \\
\hline $4 \mathrm{f}^{3} 5 \mathrm{~d}$ & 2 & & 30921 & & 1.336 & $49 \%\left({ }^{4} \mathrm{~F}^{\circ}\right) 5 \mathrm{~d}{ }^{5} \mathrm{P}^{\circ}+16 \%\left({ }^{4} \mathrm{~F}^{\circ}\right) 5 \mathrm{~d}^{3} \mathrm{~F}^{\circ}+10 \%\left({ }^{4} \mathrm{G}^{\circ}\right) 5 \mathrm{~d}{ }^{3} \mathrm{~F}^{\circ}$ \\
\hline $4 f^{3} 5 d$ & 6 & & 30931 & & 1.069 & $38 \%\left({ }^{2} \mathrm{H}^{\circ}\right) 5 \mathrm{~d}{ }^{3} \mathrm{~K}^{\circ}+28 \%\left({ }^{4} \mathrm{~F}^{\circ}\right) 5 \mathrm{~d}{ }^{5} \mathrm{G}^{\circ}+6 \%\left({ }^{4} \mathrm{~F}^{\circ}\right) 5 \mathrm{~d}{ }^{5} \mathrm{H}^{\circ}$ \\
\hline $4 f^{3} 5 d$ & 3 & & 30989 & & 1.329 & $38 \%\left({ }^{4} \mathrm{~F}^{\circ}\right) 5 \mathrm{~d}{ }^{5} \mathrm{P}^{\circ}+13 \%\left({ }^{2} \mathrm{H}^{\circ}\right) 5 \mathrm{~d}^{3} \mathrm{~F}^{\circ}+11 \%\left({ }^{4} \mathrm{~F}^{\circ}\right) 5 \mathrm{~d}{ }^{3} \mathrm{~F}^{\circ}$ \\
\hline $4 f^{3} 6 s$ & 5 & & 31268 & & 0.924 & $46 \%\left({ }^{4} \mathrm{I}^{\circ}\right) 6 \mathrm{~s}{ }^{5} \mathrm{I}^{\circ}+18 \%\left({ }^{4} \mathrm{I}^{\circ}\right) 6 \mathrm{~s}{ }^{3} \mathrm{I}^{\circ}+12 \%\left({ }^{2} \mathrm{H}^{\circ}\right) 5 \mathrm{~d}{ }^{3} \mathrm{I}^{\circ}$ \\
\hline $4 f^{3} 5 d$ & 3 & & 31371 & & 1.247 & $33 \%\left({ }^{4} \mathrm{~F}^{\circ}\right) 5 \mathrm{~d}{ }^{5} \mathrm{~F}^{\circ}+20 \%\left({ }^{4} \mathrm{~F}^{\circ}\right) 5 \mathrm{~d}{ }^{5} \mathrm{P}^{\circ}$ \\
\hline $4 f^{3} 5 d$ & 6 & 31394.6 & 31378 & 17 & 1.211 & $70 \%\left({ }^{4} \mathrm{~F}^{\circ}\right) 5 \mathrm{~d}{ }^{5} \mathrm{H}^{\circ}+12 \%\left({ }^{4} \mathrm{~F}^{\circ}\right) 5 \mathrm{~d}{ }^{5} \mathrm{G}^{\circ}+7 \%\left({ }^{4} \mathrm{I}^{\circ}\right) 5 \mathrm{~d}{ }^{5} \mathrm{H}^{\circ}$ \\
\hline $4 f^{3} 5 d$ & 2 & & 31639 & & 1.053 & $17 \%\left({ }^{4} \mathrm{~S}^{\circ}\right) 5 \mathrm{~d}{ }^{3} \mathrm{D}^{\circ}+13 \%\left({ }^{4} \mathrm{~F}^{\circ}\right) 5 \mathrm{~d}{ }^{3} \mathrm{~F}^{\circ}+13 \%\left({ }^{4} \mathrm{~F}^{\circ}\right) 5 \mathrm{~d}{ }^{3} \mathrm{P}^{\circ}$ \\
\hline $4 f^{3} 5 d$ & 3 & & 31733 & & 1.220 & $53 \%\left({ }^{4} \mathrm{~F}^{\circ}\right) 5 \mathrm{~d}{ }^{5} \mathrm{~F}^{\circ}+9 \%\left({ }^{4} \mathrm{~S}^{\circ}\right) 5 \mathrm{~d}^{3} \mathrm{D}^{\circ}$ \\
\hline $4 f^{3} 5 d$ & 7 & & 31860 & & 1.125 & $36 \%\left({ }^{2} \mathrm{H}^{\circ}\right) 5 \mathrm{~d}{ }^{3} \mathrm{~K}^{\circ}+28 \%\left({ }^{4} \mathrm{~F}^{\circ}\right) 5 \mathrm{~d}{ }^{5} \mathrm{H}^{\circ}+7 \%\left({ }^{2} \mathrm{G}^{\circ}\right) 5 \mathrm{~d}{ }^{3} \mathrm{I}^{\circ}$ \\
\hline $4 f^{3} 5 d$ & 4 & & 31866 & & 0.907 & $27 \%\left({ }^{4} \mathrm{G}^{\circ}\right) 5 \mathrm{~d}{ }^{5} \mathrm{I}^{\circ}+14 \%\left({ }^{2} \mathrm{H}^{\circ}\right) 5 \mathrm{~d}^{3} \mathrm{G}^{\circ}+11 \%\left({ }^{2} \mathrm{H}^{\circ}\right) 5 \mathrm{~d}{ }^{1} \mathrm{G}^{\circ}$ \\
\hline $4 \mathrm{f}^{3} 5 \mathrm{~d}$ & 1 & & 31924 & & 1.083 & $35 \%\left({ }^{4} \mathrm{~S}^{\circ}\right) 5 \mathrm{~d}{ }^{5} \mathrm{D}^{\circ}+18 \%\left({ }^{4} \mathrm{~F}^{\circ}\right) 5 \mathrm{~d}{ }^{3} \mathrm{D}^{\circ}+16 \%\left({ }^{4} \mathrm{~F}^{\circ}\right) 5 \mathrm{~d}{ }^{5} \mathrm{D}^{\circ}$ \\
\hline $4 f^{3} 5 d$ & 4 & & 32137 & & 0.798 & $51 \%\left({ }^{4} \mathrm{G}^{\circ}\right) 5 \mathrm{~d}{ }^{5} \mathrm{I}^{\circ}+14 \%\left({ }^{2} \mathrm{H}^{\circ}\right) 5 \mathrm{~d}{ }^{3} \mathrm{G}^{\circ}+6 \%\left({ }^{4} \mathrm{~F}^{\circ}\right) 5 \mathrm{~d}{ }^{3} \mathrm{G}^{\circ}$ \\
\hline $4 f^{3} 5 \mathrm{~d}$ & 0 & & 32178 & & & $45 \%\left({ }^{4} \mathrm{~F}^{\circ}\right) 5 \mathrm{~d}{ }^{5} \mathrm{D}^{\circ}+39 \%\left({ }^{4} \mathrm{~S}^{\circ}\right) 5 \mathrm{~d}{ }^{5} \mathrm{D}^{\circ}+7 \%\left({ }^{2} \mathrm{P}^{\circ}\right) 5 \mathrm{~d}{ }^{3} \mathrm{P}^{\circ}$ \\
\hline $4 f^{3} 5 d$ & 3 & & 32218 & & 1.180 & $26 \%\left({ }^{4} \mathrm{~F}^{\circ}\right) 5 \mathrm{~d}{ }^{5} \mathrm{P}^{\circ}+18 \%\left({ }^{2} \mathrm{H}^{\circ}\right) 5 \mathrm{~d}^{3} \mathrm{G}^{\circ}+9 \%\left({ }^{4} \mathrm{~F}^{\circ}\right) 5 \mathrm{~d}{ }^{3} \mathrm{~F}^{\circ}$ \\
\hline $4 f^{3} 5 d$ & 2 & & 32323 & & 1.266 & $28 \%\left({ }^{4} \mathrm{~S}^{\circ}\right) 5 \mathrm{~d}{ }^{5} \mathrm{D}^{\circ}+20 \%\left({ }^{4} \mathrm{~F}^{\circ}\right) 5 \mathrm{~d}{ }^{5} \mathrm{D}^{\circ}+9 \%\left({ }^{4} \mathrm{~F}^{\circ}\right) 5 \mathrm{~d}{ }^{3} \mathrm{P}^{\circ}$ \\
\hline $4 f^{3} 5 d$ & 5 & & 32330 & & 1.147 & $23 \%\left({ }^{4} \mathrm{~F}^{\circ}\right) 5 \mathrm{~d}{ }^{5} \mathrm{~F}^{\circ}+14 \%\left({ }^{4} \mathrm{~F}^{\circ}\right) 5 \mathrm{~d}{ }^{3} \mathrm{H}^{\circ}+9 \%\left({ }^{2} \mathrm{G}^{\circ}\right) 5 \mathrm{~d}{ }^{3} \mathrm{G}^{\circ}$ \\
\hline $4 f^{3} 5 d$ & 4 & & 32336 & & 1.199 & $64 \%\left({ }^{4} \mathrm{~F}^{\circ}\right) 5 \mathrm{~d}{ }^{5} \mathrm{~F}^{\circ}+12 \%\left({ }^{4} \mathrm{G}^{\circ}\right) 5 \mathrm{~d}{ }^{5} \mathrm{I}^{\circ}+7 \%\left({ }^{4} \mathrm{~F}^{\circ}\right) 5 \mathrm{~d}{ }^{3} \mathrm{G}^{\circ}$ \\
\hline $4 f^{3} 5 d$ & 6 & & 32444 & & 1.109 & $36 \%\left({ }^{2} \mathrm{H}^{\circ}\right) 5 \mathrm{~d}{ }^{1} \mathrm{I}^{\circ}+35 \%\left({ }^{4} \mathrm{~F}^{\circ}\right) 5 \mathrm{~d}{ }^{5} \mathrm{G}^{\circ}+11 \%\left({ }^{2} \mathrm{H}^{\circ}\right) 5 \mathrm{~d}{ }^{3} \mathrm{~K}^{\circ}$ \\
\hline $4 f^{3} 5 d$ & 2 & & 32589 & & 1.015 & $39 \%\left({ }^{2} \mathrm{H}^{\circ}\right) 5 \mathrm{~d}{ }^{3} \mathrm{~F}^{\circ}+15 \%\left({ }^{4} \mathrm{~F}^{\circ}\right) 5 \mathrm{~d}{ }^{5} \mathrm{D}^{\circ}+13 \%\left({ }^{4} \mathrm{~S}^{\circ}\right) 5 \mathrm{~d}{ }^{5} \mathrm{D}^{\circ}$ \\
\hline $4 f^{3} 6 s$ & 6 & & 32792 & & 1.060 & $64 \%\left({ }^{4} \mathrm{I}^{\circ}\right) 6 \mathrm{~s}{ }^{5} \mathrm{I}^{\circ}+18 \%\left({ }^{4} \mathrm{I}^{\circ}\right) 6 \mathrm{~s}^{3} \mathrm{I}^{\circ}+7 \%\left({ }^{2} \mathrm{H}^{\circ}\right) 5 \mathrm{~d}{ }^{3} \mathrm{I}^{\circ}$ \\
\hline $4 f^{3} 5 d$ & 7 & 32832.6 & 32818 & 15 & 1.178 & $53 \%\left({ }^{4} \mathrm{~F}^{\circ}\right) 5 \mathrm{~d}{ }^{5} \mathrm{H}^{\circ}+28 \%\left({ }^{2} \mathrm{H}^{\circ}\right) 5 \mathrm{~d}{ }^{3} \mathrm{~K}^{\circ}$ \\
\hline $4 f^{3} 5 d$ & 1 & & 32830 & & 1.121 & $26 \%\left({ }^{4} \mathrm{~F}^{\circ}\right) 5 \mathrm{~d}{ }^{5} \mathrm{D}^{\circ}+19 \%\left({ }^{4} \mathrm{~S}^{\circ}\right) 5 \mathrm{~d}{ }^{3} \mathrm{D}^{\circ}$ \\
\hline $4 f^{3} 5 d$ & 4 & & 32848 & & 1.217 & $40 \%\left({ }^{4} \mathrm{~F}^{\circ}\right) 5 \mathrm{~d}{ }^{3} \mathrm{~F}^{\circ}+19 \%\left({ }^{4} \mathrm{G}^{\circ}\right) 5 \mathrm{~d}{ }^{3} \mathrm{~F}^{\circ}$ \\
\hline $4 f^{3} 6 s$ & 5 & & 32959 & & 0.962 & $34 \%\left({ }^{4} \mathrm{I}^{\circ}\right) 6 \mathrm{~s}{ }^{3} \mathrm{I}^{\circ}+17 \%\left({ }^{4} \mathrm{I}^{\circ}\right) 6 \mathrm{~s}{ }^{5} \mathrm{I}^{\circ}+13 \%\left({ }^{4} \mathrm{G}^{\circ}\right) 5 \mathrm{~d}{ }^{5} \mathrm{I}^{\circ}$ \\
\hline
\end{tabular}

a Experimental energy levels taken from Martin et al. (1978).

b Calculated energy levels obtained in the present work.

c $\Delta E=E_{\exp }-E_{\text {calc }}$.

d Landé $g$-factors calculated in the present work.

e Only the three major components larger than $5 \%$ are given.

line strengths are affected by severe cancellation effects. Indeed, the cancellation factor $(C F)$, as defined by Cowan (1981), is generally smaller than 0.01 in both calculations for these two transitions. For the lines at 353.7622 and $370.1320 \mathrm{~nm}$, the discrepancies between our calculated oscillator strengths and Bord's results could be due to the fact that the upper $4 \mathrm{f}^{3} 5 \mathrm{~d}$ level, situated at $29397.3 \mathrm{~cm}^{-1}$, is strongly mixed $\left(31 \%\left({ }^{4} \mathrm{~F}^{\circ}\right) 5 \mathrm{~d}^{5} \mathrm{H}^{\circ}+14 \%\left({ }^{2} \mathrm{H}^{\circ}\right) 5 \mathrm{~d}^{3} \mathrm{I}^{\circ}+\right.$ $12 \%\left({ }^{4} \mathrm{~F}^{\circ}\right) 5 \mathrm{~d}^{5} \mathrm{G}^{\circ}$ in our calculation) and is probably very sensitive to small differences in the eigenvector compositions.

For the reasons stated above, the present set of transition probabilities is expected to be the most accurate presently available. The detailed results are available in the database mentioned at the end of Sect. 1 .

\section{Nd III in stellar spectra}

The information concerning the occurence of the third spectra of the REE in stellar sources is rather scarse and scattered through the literature although the presence of these ions in stellar spectra has long been recognized (Swings 1944; Adelman 1974; Cowley 1976). Some systematic searches have nevertheless been undertaken (see e.g. Cowley \& Greenberg 1988) and, more recently, the satellite spectra have stimulated new investigations of these ions: Copernicus spectra of $\alpha^{2} \mathrm{CVn}$ or BUSS spectra of the same star have been investigated by Leckrone (1976) and by Hensberge et al. (1986), respectively. IUE spectra of HD 51418 have been considered by Adelman \& Shore (1981). 
Table 5. Calculated oscillator strengths $(\log g f)$ and transition probabilities $(g A)$ for $4 \mathrm{f}^{4}-4 \mathrm{f}^{3} 5 \mathrm{~d}$ transitions of Nd III. Only transitions for which $\log g f>-4.0$ are given in the table. Wavelengths in air are deduced from the experimental energy levels compiled by Martin et al. (1978). For each level, we give the energy $\left(\mathrm{cm}^{-1}\right)$, the parity (o: odd; e: even) and the $J$ value.

\begin{tabular}{|c|c|c|c|c|c|c|c|c|c|}
\hline \multirow{2}{*}{$\frac{\lambda(\mathrm{nm})}{328.367}$} & \multicolumn{3}{|c|}{ Lower level } & \multicolumn{3}{|c|}{ Upper level } & \multirow{2}{*}{$\frac{\log g f}{-3.25}$} & \multirow{2}{*}{$\frac{\mathrm{gA}(1)}{3.50 \mathrm{E}+05}$} & \multirow{2}{*}{$\frac{\mathrm{gA}(2)}{3.94 \mathrm{E}+05}$} \\
\hline & 2387.6 & (e) & 6.0 & 32832.6 & (o) & 7.0 & & & \\
\hline 330.409 & 1137.8 & (e) & 5.0 & 31394.6 & (o) & 6.0 & -3.30 & $3.06 \mathrm{E}+05$ & $3.54 \mathrm{E}+05$ \\
\hline 330.677 & 0.0 & (e) & 4.0 & 30232.3 & (o) & 5.0 & -3.35 & $2.70 \mathrm{E}+05$ & $2.78 \mathrm{E}+05$ \\
\hline 343.335 & 3714.9 & (e) & 7.0 & 32832.6 & (o) & 7.0 & -1.65 & $1.28 \mathrm{E}+07$ & $1.44 \mathrm{E}+07$ \\
\hline 343.609 & 1137.8 & (e) & 5.0 & 30232.3 & (o) & 5.0 & -1.64 & $1.30 \mathrm{E}+07$ & $1.34 \mathrm{E}+07$ \\
\hline 344.646 & 2387.6 & (e) & 6.0 & 31394.6 & (o) & 6.0 & -1.64 & $1.29 \mathrm{E}+07$ & $1.49 \mathrm{E}+07$ \\
\hline 347.783 & 0.0 & (e) & 4.0 & 28745.3 & (o) & 4.0 & -1.97 & $6.00 \mathrm{E}+06$ & $3.81 \mathrm{E}+06$ \\
\hline 353.762 & 1137.8 & (e) & 5.0 & 29397.3 & (o) & 5.0 & -2.65 & $1.22 \mathrm{E}+06$ & \\
\hline 359.032 & 2387.6 & (e) & 6.0 & 30232.3 & (o) & 5.0 & -0.77 & $8.74 \mathrm{E}+07$ & $8.99 \mathrm{E}+07$ \\
\hline 359.762 & 0.0 & (e) & 4.0 & 27788.2 & (o) & 3.0 & -0.91 & $6.35 \mathrm{E}+07$ & $6.35 \mathrm{E}+07$ \\
\hline 360.397 & 5093.3 & (e) & 8.0 & 32832.6 & (o) & 7.0 & -0.62 & $1.24 \mathrm{E}+08$ & $1.40 \mathrm{E}+08$ \\
\hline 361.173 & 3714.9 & (e) & 7.0 & 31394.6 & (o) & 6.0 & -0.54 & $1.46 \mathrm{E}+08$ & $1.69 \mathrm{E}+08$ \\
\hline 362.117 & 1137.8 & (e) & 5.0 & 28745.3 & (o) & 4.0 & -0.82 & $7.68 \mathrm{E}+07$ & $4.88 \mathrm{E}+07$ \\
\hline 362.612 & 0.0 & (e) & 4.0 & 27569.8 & (o) & 3.0 & -1.20 & $3.16 \mathrm{E}+07$ & \\
\hline 370.132 & 2387.6 & (e) & 6.0 & 29397.3 & (o) & 5.0 & -1.05 & $4.38 \mathrm{E}+07$ & \\
\hline 394.126 & 1137.8 & (e) & 5.0 & 26503.2 & (o) & 6.0 & -3.75 & $7.60 \mathrm{E}+04$ & \\
\hline 414.552 & 2387.6 & (e) & 6.0 & 26503.2 & (o) & 6.0 & -2.70 & $7.83 \mathrm{E}+05$ & \\
\hline 422.241 & 3714.9 & (e) & 7.0 & 27391.4 & (o) & 8.0 & -2.74 & $6.83 \mathrm{E}+05$ & \\
\hline 438.698 & 3714.9 & (e) & 7.0 & 26503.2 & (o) & 6.0 & -2.38 & $1.46 \mathrm{E}+06$ & \\
\hline 440.764 & 1137.8 & (e) & 5.0 & 23819.3 & (o) & 6.0 & -3.62 & $8.29 \mathrm{E}+04$ & \\
\hline 448.343 & 5093.3 & (e) & 8.0 & 27391.4 & (o) & 8.0 & -1.34 & $1.51 \mathrm{E}+07$ & \\
\hline 456.768 & 0.0 & (e) & 4.0 & 21886.8 & (o) & 5.0 & -3.28 & $1.66 \mathrm{E}+05$ & \\
\hline 462.499 & 2387.6 & (e) & 6.0 & 24003.2 & (o) & 7.0 & -1.98 & $3.33 \mathrm{E}+06$ & \\
\hline 466.468 & 2387.6 & (e) & 6.0 & 23819.3 & (o) & 6.0 & -3.28 & $1.62 \mathrm{E}+05$ & \\
\hline 476.704 & 3714.9 & (e) & 7.0 & 24686.4 & (o) & 8.0 & -1.71 & $5.65 \mathrm{E}+06$ & \\
\hline 478.106 & 1137.8 & (e) & 5.0 & 22047.8 & (o) & 6.0 & -1.69 & $6.03 \mathrm{E}+06$ & \\
\hline 490.326 & 0.0 & (e) & 4.0 & 20388.9 & (o) & 5.0 & -1.83 & $4.13 \mathrm{E}+06$ & \\
\hline 492.102 & 2387.6 & (e) & 6.0 & 22702.9 & (o) & 7.0 & -1.77 & $4.66 \mathrm{E}+06$ & \\
\hline 492.757 & 3714.9 & (e) & 7.0 & 24003.2 & (o) & 7.0 & -0.83 & $4.08 \mathrm{E}+07$ & \\
\hline 496.280 & 0.0 & (e) & 4.0 & 20144.3 & (o) & 4.0 & -3.45 & $9.53 \mathrm{E}+04$ & \\
\hline 497.265 & 3714.9 & (e) & 7.0 & 23819.3 & (o) & 6.0 & -1.26 & $1.52 \mathrm{E}+07$ & \\
\hline 508.500 & 2387.6 & (e) & 6.0 & 22047.8 & (o) & 6.0 & -0.66 & $5.71 \mathrm{E}+07$ & \\
\hline 510.242 & 5093.3 & (e) & 8.0 & 24686.4 & (o) & 8.0 & -0.38 & $1.05 \mathrm{E}+08$ & \\
\hline 512.699 & 2387.6 & (e) & 6.0 & 21886.8 & (o) & 5.0 & -1.08 & $2.11 \mathrm{E}+07$ & \\
\hline 519.306 & 1137.8 & (e) & 5.0 & 20388.9 & (o) & 5.0 & -0.75 & $4.42 \mathrm{E}+07$ & \\
\hline 520.390 & 0.0 & (e) & 4.0 & 19211.0 & (o) & 3.0 & -1.19 & $1.60 \mathrm{E}+07$ & \\
\hline 525.989 & 1137.8 & (e) & 5.0 & 20144.3 & (o) & 4.0 & -1.12 & $1.83 \mathrm{E}+07$ & \\
\hline 526.502 & 3714.9 & (e) & 7.0 & 22702.9 & (o) & 7.0 & -0.66 & $5.30 \mathrm{E}+07$ & \\
\hline 528.676 & 5093.3 & (e) & 8.0 & 24003.2 & (o) & 7.0 & -1.51 & $7.41 \mathrm{E}+06$ & \\
\hline 529.410 & 0.0 & (e) & 4.0 & 18883.7 & (o) & 4.0 & -0.65 & $5.23 \mathrm{E}+07$ & \\
\hline 545.316 & 3714.9 & (e) & 7.0 & 22047.8 & (o) & 6.0 & -3.85 & $3.19 \mathrm{E}+04$ & \\
\hline 563.354 & 1137.8 & (e) & 5.0 & 18883.7 & (o) & 4.0 & -2.06 & $1.82 \mathrm{E}+06$ & \\
\hline 567.715 & 5093.3 & (e) & 8.0 & 22702.9 & (o) & 7.0 & -1.41 & $8.02 \mathrm{E}+06$ & \\
\hline 584.507 & 5093.3 & (e) & 8.0 & 22197.0 & (o) & 9.0 & -1.13 & $1.44 \mathrm{E}+07$ & \\
\hline 598.780 & 3714.9 & (e) & 7.0 & 20410.9 & (o) & 8.0 & -1.22 & $1.13 \mathrm{E}+07$ & \\
\hline 614.507 & 2387.6 & (e) & 6.0 & 18656.3 & (o) & 7.0 & -1.29 & $9.05 \mathrm{E}+06$ & \\
\hline 632.724 & 1137.8 & (e) & 5.0 & 16938.1 & (o) & 6.0 & -1.36 & $7.31 \mathrm{E}+06$ & \\
\hline 652.664 & 5093.3 & (e) & 8.0 & 20410.9 & (o) & 8.0 & -2.36 & $6.77 \mathrm{E}+05$ & \\
\hline 655.033 & 0.0 & (e) & 4.0 & 15262.2 & (o) & 5.0 & -1.44 & $5.73 \mathrm{E}+06$ & \\
\hline 669.097 & 3714.9 & (e) & 7.0 & 18656.3 & (o) & 7.0 & -2.31 & $7.21 \mathrm{E}+05$ & \\
\hline 687.072 & 2387.6 & (e) & 6.0 & 16938.1 & (o) & 6.0 & -2.44 & $5.13 \mathrm{E}+05$ & \\
\hline 707.799 & 1137.8 & (e) & 5.0 & 15262.2 & (o) & 5.0 & -2.73 & $2.53 \mathrm{E}+05$ & \\
\hline
\end{tabular}

$g A(1)$ : Weighted HFR transition probabilities (this work).

$g A(2)$ : Weighted HFR transition probabilities normalized with the laser measurements of the present work. 


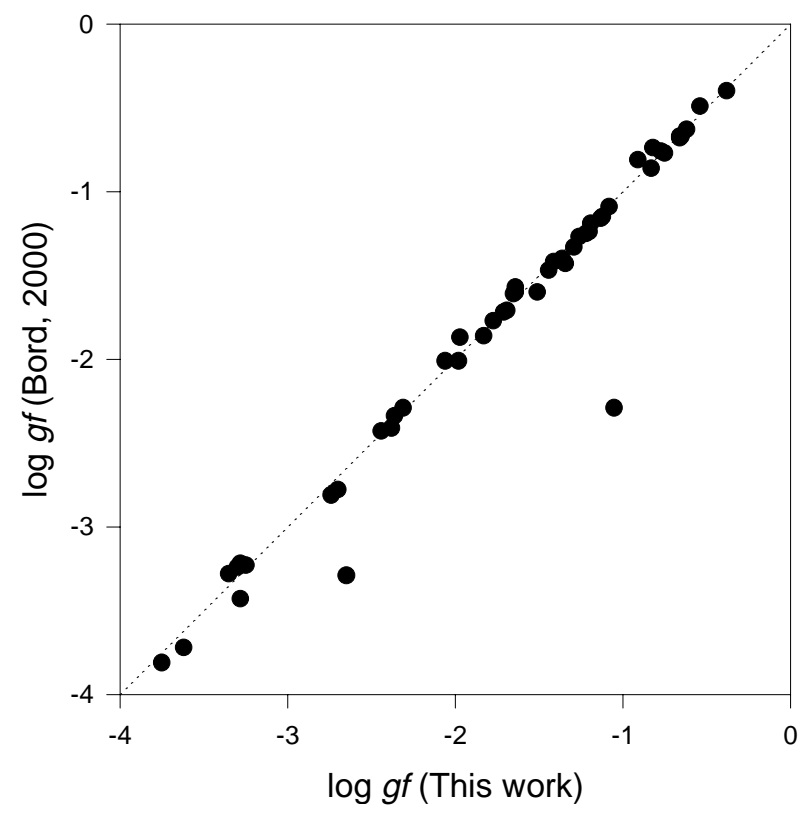

Fig. 3. Comparison of the HFR oscillator strengths obtained in this work with those published by Bord (2000).

Although Nd II is expected to be the dominant species in cold CP stars, it is important to compare the abundances derived from this ion to those obtained from the consideration of Nd III lines in order to get information on eventual non-LTE effects or on possible element stratification in the stellar atmospheres (Proffitt et al. 1999). In addition, the lines of doubly ionized REE are expected to be stronger in hotter stars where the doubly ionized elements could be dominant but the identification of the lines and the abundance work, in many cases, has been performed in the past or is still prevented by the lack of reliable atomic data.

As pointed out in the introduction, Nd III has been identified or mentioned as possibly present in a number of stars: HR 465, HD 51418, HD 200311 (Cowley 1976; Aikman et al. 1979; Bidelman et al. 1995), HD 192913 (Cowley \& Crosswhite 1978; Ryabchikova et al. 1990), HD 101065 (Cowley et al. 1998) but the identification can be complicated by problems of different types. The identification of lanthanides in stars showing strong Si II lines is made difficult by the fact that only a few stars are known which show very sharp lines. According to Cowley (1976), Nd III is strong in the spectrum of HR 465 but, in $\beta \mathrm{CrB}$, the coincidences are indicated as marginally significant, the author insisting however upon the fact that the strong Nd III lines appear shortward of the Balmer jump and, consequently, that his study is not well suited for the detection of this ion.

For HD 101065, Bord (2000) has deduced an abundance of neodymium 5000 larger than the solar result, a value similar to that deduced from an analysis of $\mathrm{Nd}$ II lines; this result has to be reduced by $0.7-0.8$ dex if the surface magnetic field is taken into account. The analysis by
Ryabchikova et al. (2000) of the cool roAp star HD 122970 , based on the $g f$-values of Bord (2000), leads to an overabundance of $\mathrm{Nd}$ by a factor of about 275 relative to the solar abundance. We did not try to correct the results of these two analyses on the basis of the new $g f$-values obtained in the present work because the detailed list of the transitions considered is not available in the corresponding papers. Nevertheless, on the basis of the general agreement of the two sets of data observed in Fig. 1, the general conclusions of Bord (2000) remain valid. The main improvement due to the present work is the fact that these results are now firmly established on the basis of the laser experimental results.

In conclusion, as an extrapolation of the present work, it is expected that, in the future, the new transition probabilities now available for Nd III will make possible more reliable quantitative analysis of stellar spectra of CP stars.

Acknowledgements. This work was financially supported by the Swedish Natural Science Research Council and by the EU-TMR access to Large-Scale facility programme (contract HPRI-CT-1999-00041). Financial support from the Belgian FNRS is acknowledged by E.B., P.P. and P.Q.

\section{References}

Adelman, S. J. 1973, ApJS, 26, 1

Adelman, S. J. 1974, ApJS, 28, 51

Adelman, S. J., \& Shore, S. N. 1981, PASP, 93, 85

Aikman, G. C. L., Cowley, C. R., \& Crosswhite, H. 1979, ApJ, 232,812

Anders, E., \& Grevesse, N. 1989, Geoch. Cosmochim. Acta, 53, 197

Bidelman, W. P. 1953, ApJ, 117, 377

Bidelman, W. P., Cowley, C. R., \& Iler, A. L. 1995, Publ. Obs. Univ. Michigan, 12, 77

Biémont, E., Li, Z. S., Palmeri, P., \& Quinet, P. 1999, J. Phys. B, 32, 3409

Biémont, E., Garnir, H. P., Bastin, T., et al. 2001a, MNRAS, 321,481

Biémont, E., Garnir, H. P., Palmeri, P., et al. 2001b, Phys. Rev., 64, 022503

Biémont, E., Garnir, H. P., Li, Z. S., et al. 2001c, J. Phys. B, 34,1869

Biémont, E., Palmeri, P., Quinet, P., et al. 2001d, MNRAS, 328,1085

Bord, D. J. 2000, A\&A, 144, 517

Brewer, J. 1971, JOSA, 59, 2083

Cowan, R. D. 1981, The theory of Atomic Structure and Spectra (University of California Press, Berkeley, CA, USA)

Cowley, C. R. 1976, ApJS, 32, 631

Cowley, C. R. 1984, Phys. Scr., T8, 28

Cowley, C. R., \& Bord, D. J. 1998, The Third Spectrum of Neodymium: Application to HR 6870 and $\gamma$ Equulei, in The Scientific Impact of the Goddard High Resolution Spectrograph, ed. J. C. Brandt, T. B. Ake III, C. C. Petersen, ASP Conf. Ser., 143, 346

Cowley, C. R., \& Crosswhite, H. M. 1978, PASP, 90, 108

Cowley, C. R., Bidelman, W. P., \& Mathys, G. 1998, BAAS, 30, 1317

Cowley, C. R., \& Greenberg, M. 1988, MNRAS, 232, 763 
Cowley, C. R., Ryabchikova, T., Kupka, F., et al. 2000, MNRAS, 317, 299

Crosswhite, H. 1975, 1976, unpublished work, quoted by Martin et al. (1978)

Hensberge, H., Van Santvoort, J., Van der Hucht, K. A., \& Morgan, T. H. 1986, A\&A, 158, 113

Jaschek, C., \& Jaschek, M. 1995, The Behavior of Chemical Elements in Stars Cambridge University Press)

Lambert, D. L. 1985, in Cool Stars with Excesses of Heavy Elements, ed. M. Jaschek, \& P. C. Keenan (Reidel), 191

Leckrone, D. S. 1976, in The Physics of Ap Stars, IAU Colloq. 32, ed. W. W. Weiss, H. Jenkner, \& J. J. Wood, Universitätssterwarte Wien, Vienna

Leckrone, D. S., Proffitt, C. R., Wahlgren, G. M., Johansson, S. G., \& Brage, T. 1999, AJ., 117, 1454

Li, Z. S., Lundberg, H., Wahlgren ,G. M., \& Sikström, C. M. 2000, Phys. Rev. A, 62, Electronic version Ref. No. 032505

Li, Z. S., Zhang, Z. G., Lokhnygin, V., et al. 2001, J. Phys. B, 34,1349

Li, Z. S., \& Jiang, Z. K. 1999, Phys. Scr., 60, 414

Martin, W. C., Zalubas, R., \& Hagan, L. 1978, Atomic Energy Levels, The Rare-Earth Elements, Nat. Bur. Stand. Ref. Data Ser. 60, Washington D.C., USA
Merrill P.W. 1956, Lines of the Chemical Elements in Astronomical Spectra, Carnegie Institute of Washington, Publ., 610

Palmeri, P., Quinet, P., Frémat, Y., Wyart, J.-F., \& Biémont, E. 2000, ApJS, 129, 367

Proffitt, C. R., Brage, T., Leckrone, D. S., et al. 1999, ApJ, 512, 942

Quinet, P., Palmeri, P., Biémont, E., et al. 1999, MNRAS, 307, 934

Ryabchikova, T. A., Davidova, E. S., \& Adelman, S. J. 1990, PASP, 102, 581

Ryabchikova, T. A., Savanov, I. S., Hatzes, A. P., Weiss, W. W., \& Handler, G. 2000, A\&A, 357, 981

Smith, M. A. 1973, ApJS, 25, 277

Smith, M. A. 1974, ApJ, 189, 101

Sneden, C., McWilliam, A., Preston, G. W., et al. 1996, ApJ, 467,819

Smith, V. V., \& Lambert, D. L. 1985, ApJ, 294, 326

Swings, P. 1944, ApJ, 100, 132

Zhang, Z. G., Li, Z. S., Lundberg, H., et al. 2000, J. Phys. B, 33,521

Zhang, Z. G., Persson, A., Li, Z. S., Svanberg, S., \& Jiang, Z. K. 2001, Eur. Phys. J. D, 13, 301 